\title{
Plasma purification is associated with reduced inflammation factors and down-regulation of lipid metabolic and ER stress proteins in patients with hyperlipidemia
}

\section{Xiao Meng Zhang}

Division of Nephrology, Shanghai Pudong Hospital, Fudan University

Yan Hong Gu

Shanghai Pudong Hospital

Hao Deng

Division of Nephrology, Shanghai Pudong Hospital, Fudan University

\section{Zheng Quan Xu}

The First Affiliated Hospital of Medical School of Zhejiang University

\section{Ze Yuan Zhong}

Division of Orthopedic, Shanghai Pudong Hospital, Fudan University

Hui Min Jin ( $\square$ hmjgli@163.com )

Shanghai Pudong Hospital, Fudan University,

\section{Xiu Hong Yang}

Division of Nephrology, Shanghai Pudong Hospital, Fudan University

\section{Research}

Keywords: Lipid protein, double-filtration plasmapheresis (DFPP), endoplasmic reticulum (ER) stress, apoptosis, peripheral blood mononuclear cells (PBMCs), reactive oxygen species (ROS), apoptosis-related proteins, inflammatory factors

Posted Date: November 17th, 2020

DOI: https://doi.org/10.21203/rs.3.rs-70758/v3

License: (c) (1) This work is licensed under a Creative Commons Attribution 4.0 International License. Read Full License 


\section{Abstract}

\section{Background}

Hyperlipidaemia is a strong risk factor for arteriosclerosis and cardiovascular disease (CVD). Therapy with lipid-lowering drugs often results in many side effects. The aim of our study was to investigate the potential effects of non-drug therapy with double-filtration plasmapheresis (DFPP) on lipid metabolism-, endoplasmic reticulum (ER) stress- and apoptosis-related proteins in peripheral blood mononuclear cells (PBMCs) before and after lipid clearance in patients with hyperlipidaemia.

\section{Methods}

Thirty-five patients with hyperlipidaemia were selected for this study. Proteins related to lipid metabolism [CD36, proprotein convertase subtilisin/kexin type 9 (PCSK9) and LDL receptor], ER stress [glucoseregulated protein 78 (GRP78), C/EBP homoiogousprotein (CHOP), activating transcription factor 4 (ATF4), eukaryotic initiation factor 2 (EIF2a)] and apoptosis (Bcl-2, BAX, and Caspase-3) were assayed by western blot, reactive oxygen species (ROS) were measured by flow cytometry (FCM), and serum inflammatory factors were detected by ELISA.

\section{Results}

Compared with their pre-DFPP values, the values of most lipid metabolic parameters, such as cholesterol, triglycerides, LDL, lipoprotein a [Lp(a)] and small dense LDL cholesterol (sdLDL), were reduced after DFPP. DFPP was associated with the downregulation of proteins related to lipid metabolism, ER stress and apoptosis, which also resulted in decreased ROS and serum inflammatory factor release.

\section{Conclusion}

DFPP has lipid-lowering activity and can also regulate lipid metabolism-, ER stress- and apoptosis-related proteins in PBMCs and reduce the levels of inflammatory factors in patients with hyperlipidaemia (ClinicalTrials.gov number: NCT03491956).

\section{Introduction}

Hyperlipidaemia is one of the most important factors associated with cardiovascular disease (CVD) and often results in fatty liver, cerebral thrombosis and/or infarction, and severe pancreatitis [1-4]. However, there is low awareness of the risk of CVD, and the benefits of treatment are underappreciated in the general population. Lipid disorders are present in $40.4 \%$ of the adult population in China, with hypercholesterolemia present in $4.9 \%$ of the population, hypertriglyceridemia present in $13.1 \%$ of the population, and high-low-density lipoproteinemia present in $33.9 \%$ of the population [5]. Therefore, there is an urgent need for prevention and treatment of increased serum lipid concentrations. 
One important marker of hyperlipidaemia is abnormal expression of lipid-related metabolic proteins. CD36 is a lipid transporter and multifunctional scavenger receptor [6]. It plays roles in modification of LDL and uptake of modified lipoproteins, which causes macrophage lipid accumulation, leading to the formation of foam cells [7]. In hyperlipidaemia patients, CD36 expression was abnormal, and increased CD36 expression could result in endoplasmic reticulum (ER) stress, macrophage apoptosis, insulin resistance and CVD [8]. Another important lipid metabolic protein, proprotein convertase subtilisin/kexin type 9 (PCSK9), is mainly expressed in the liver, intestinal tract and kidney. In the context of decreased PCSK9 activity, LDL receptor (LDLR) is upregulated, resulting in a decrease in LDL cholesterol levels, potentially reducing the risk of CVD [9]. Previous studies have indicated that PCSK9 levels were associated with metabolism-related indices, such as fasting blood glucose, insulin, triglycerides and liver triglyceride content [10]. Alirocumab, a PCSK9 inhibitor, has been approved by the US Food and Drug Administration in the treatment of patients with hypercholesterolemia. In another study, Alirocumab was found to reduce or improve cardiovascular outcomes after acute coronary syndrome [11]. Previous studies have indicated that PCSK9 and CD36 are associated with lipid metabolism and CVD outcomes [12]. However, it is unclear whether lipid plasmapheresis could affect CD36, PCSK9 and LDLR.

Statins are the most frequently used drug for the treatment of hypercholesterolemia. However, adverse reactions, such as rhabdomyolysis, abnormal liver enzymes and gastrointestinal symptoms, frequently prevent long-term use of this medication. Moreover, a meta-analysis indicated that statins were associated with an increased risk of insulin resistance and new-onset diabetes [13]. Similarly, Ezetimibe shows the same side effects as statins [14]. Some patients are reluctant to take lipid-lowering drugs considering the adverse effects of statins, and some are worried about control of blood glucose, especially among patients with diabetes.

Non-drug therapy to reduce lipid levels has been used for many years. Heparin-induced extracorporeal LDL precipitation (HELP) was proven to be capable of reducing lipid levels and the risk of CVD in patients with familial hypercholesterolemia in a previous study; however, the high costs of consumption and treatment prevented its further large-scale clinical usage. Instead, double-filtration plasmapheresis (DFPP) has recently replaced HELP in the treatment of familial hypercholesterolemia, hyperlipidaemiainduced severe acute pancreatitis, and coronary artery disease after coronary artery stenosis [15-18]. DFPP has the advantages of simple use for operators, safety for patients, a low price (7000 RMB, equal to 1000 US dollars/session, can be covered by Medicare), a short treatment time (3-4 hours) and no plasma or albumin replacement.

DFPP technology has been used to treat hyperlipidaemia patients at Pudong Medical Center since 2018. The aim of this study was to investigate the lipid removal effect of DFPP and the association of DFPP with changes in lipid metabolism- and ER stress-related proteins in PBMCs before and after treatment in patients with hyperlipidaemia.

\section{Materials And Methods}




\section{Study participants}

Patients who had hyperlipidaemia [diagnosed with hypercholesterolemia (cholesterol $>5.18 \mathrm{mmol} / \mathrm{L}$ ) or hypertriglyceridemia (triglycerides $>2.3 \mathrm{mmol} / \mathrm{L}$ ), mixed hyperlipidaemia (cholesterol $>5.18 \mathrm{mmol} / \mathrm{L}$ and triglycerides $>2.3 \mathrm{mmol} / \mathrm{L}$ ), and high low-density lipoproteinemia (low-density lipoprotein $>3.4 \mathrm{mmol} / \mathrm{L}$ ) according to the 2019 guidelines of the European Society of Cardiology] with no other diseases were selected for this study. No statins or other lipid-lowering drugs were prescribed for the patients. All patients provided and signed informed consent. The study was approved by the Ethical Committee of Shanghai Pudong Hospital (Fudan University, Pudong Medical Center, Shanghai, China), and experiments were performed in accordance with the Ethical Committee's guidelines and regulations. The ClinicalTrials.gov number was NCT 03491956.

Exclusion criteria: Patients were excluded if (1) they had coexisting diabetes or hypertension; (2) they had tumours, liver and kidney dysfunction, severe oedema, cardiac insufficiency, respiratory insufficiency caused by severe lung disease, or pregnancy; and (3) they were over 80 years old.

\section{Treatment procedure}

Plasmapheresis using a Plasauto iQ automatic blood purification system, model KM-9000 (Kawasumi, Tokyo, Japan), was performed in this study, with a PE-08 primary membrane plasma separator and an EC-4A20 secondary membrane plasma component separator. Cubital elbow median veins on both arms were selected for artery and vein access. The blood flow was $60-100 \mathrm{~mL} / \mathrm{min}$, the plasma separation rate was $30 \%$ of the blood flow, and the plasma rejection rate was $15 \%$ of the plasma separation rate. Ordinary heparin was used as an anticoagulant, with an initial dose of $3000 \mathrm{U}$, followed by an additional maintenance dose of $20-40 \mathrm{U} /(\mathrm{kg} \cdot \mathrm{h})$. The therapeutic target was calculated as weight $(\mathrm{kg}){ }^{\prime} 40 \mathrm{~mL}(1-1.4$ times blood volume). Each session lasted for 3-4 hours.

\section{Laboratory examination}

\section{Isolation of PBMCs}

Blood samples were taken from patients with hyperlipidaemia, and acidic citrate dextrose (Walvax, Yunnan, China) was added as an anticoagulant. To isolate PBMCs, the blood samples were diluted with PBS, and lymphocyte separation medium was added (Hao Yang Biological Manufacture Co., Tianjin, China). The samples were centrifuged at $1000 \mathrm{rpm}$ for $40 \mathrm{~min}$, the middle cell layer was collected, and PBMCs were obtained after washing three times with PBS.

\section{Detection of lipid metabolism-related, ER stress-related and apoptosis-related proteins}

The proteins subjected to western blotting were extracted using lysis buffer (Invent, Beijing, China), separated by sodium dodecyl sulfate-polyacrylamide gel electrophoresis (SDS-PAGE) and transferred onto polyvinylidene difluoride (PVDF) membranes (Millipore, MA, USA). The membrane carrying the protein bands was blocked in Tris-buffered saline with Tween (TBST) containing 5\% skim milk for 1 hour 
and incubated with primary antibodies at $4{ }^{\circ} \mathrm{C}$ overnight. After washing, the membrane was incubated with secondary antibodies conjugated to horseradish peroxidase for 1 hour at room temperature. Secondary antibodies included goat anti-rabbit lgG and goat anti-mouse lgG. After washing, the membranes were incubated with Immobilon Western Chemiluminescent HRP Substrate (Millipore, MA, USA). Protein signals were captured using a Bio-Rad ChemiDoc ${ }^{\mathrm{TM}}$ XRS system (Bio-Rad, CA, USA). Data were quantified by Quantity One software (Bio-Rad, CA, USA) [19]. Primary antibodies against LDLR, CD36, PCSK9, GRP78, CHOP, ATF4, p-EIF2, Bcl-2, BAX and Caspase-3 and secondary antibodies were obtained from Proteintech (Proteintech Group, Inc., IL, USA), and an antibody against EIF2 was purchased from CST (Cell Signaling Technology, MA, USA). The antibodies are diluted according to the instructions, usually 1:2000-5000.

\section{Detection of ROS and serum inflammatory factors}

Nonstimulated PBMCs were detected with a ROS assay kit (Beyotime, Shanghai, China) after isolation. PBMCs in each sample were washed twice with PBS. The fluorescent probe used to determine the level of ROS in PBMCs was diluted to a concentration of 10 umol/L. PBMCs were suspended in the diluted fluorescent probes and incubated in a plate at $37^{\circ} \mathrm{C}$ for $20 \mathrm{~min}$. The PBMCs were washed three times with cell culture medium to remove probe that did not enter the cells. Finally, the ROS level was detected by flow cytometry (BD, NH, USA). Serum inflammatory factors [Interleukin 1b (IL-1b)凶 Interleukin 6 (IL-6), Tumor necrosis factor a (TNF-a)] were detected by ELISA using commercial kits (Neobioscience, Shanghai, China).

\section{Statistical analysis}

Data are expressed as the means \pm SDs, with the exception of skewed data, which are expressed as the median (range). After correcting non-normally distributed data, the differences were analysed with a paired $\mathrm{t}$ test, and a $P$ value $<0.05$ was considered statistically significant. Statistical analyses were performed using SPSS 22.0 statistical software (IBM, IL, USA).

\section{Results}

\section{Clinical characteristics of the patients}

The age, sex, weight and baseline lipid profiles of the patients are shown in Table 1. The mean serum total cholesterol post DFPP was $2.4[1.8,3.1] \mathrm{mM}$, which represented a significant decrease compared to the pre-DFPP values. Serum LDL and triglycerides post DFPP showed the same trend. Compared with the values measured before DFPP, Lp(a) and sdLDL values also showed an obvious decrease post DFPP. These data indicated that DFPP could remove blood lipid molecules effectively in 3-4 hours.

\section{DFPP was associated with changes in lipid metabolism-related proteins}

The protein expression levels of lipid metabolism-related proteins assayed by western blot are shown in

Fig 1. LDLR, PCSK9 and CD36 were all down-regulated post DFPP. Our results indicated that DFPP may 
influence key lipid metabolism-related transport proteins.

\section{DFPP reduced the expression of ER stress-related proteins}

The expression of ER stress-related proteins (GRP78, CHOP, ATF4 and p-EIF2a) is shown in Fig 2. Compared with the expression of these proteins prior to DFPP, GRP78, CHOP, ATF4 and p-EIF2a showed the significant decreases post DFPP. These data demonstrated that DFPP was associated with reduced ER stress in hyperlipidaemia patients.

\section{DFPP inhibited hyperlipidaemia-induced cell apoptosis-related protein expression}

As Fig 3 shows, excessive lipids induced increased expression of apoptosis-related proteins in PBMCs, and DFPP treatment fully restored the expression of Bcl-2 and inhibited the expression of BAX and Caspase 3.

\section{DFPP reduced ROS levels in PBMCs and alleviated inflammatory factors in serum}

The ROS levels in hyperlipidaemia patients were significantly decreased compared with those of patients before DFPP (Fig 4). In addition, in Table 2, markers of inflammation showed an obvious decline after DFPP. These results showed that DFPP could effectively decrease ROS levels and reduce inflammatory factor release in hyperlipidaemia patients.

\section{Adverse events}

One patient experienced a hypotensive episode during the procedure and quickly recovered after infusion of $0.9 \%$ normal saline. No other adverse events (blood coagulation, bleeding, or allergy) occurred during the treatment period.

\section{Discussion}

Our study indicates that DFPP could perfectly reduce the levels of lipids, such as LDL, cholesterol, triglycerides, Lp(a), and sdLDL-cholesterol, and this finding is similar to the results of other studies [15]. The safety, easy operation and low cost of DFPP indicate that it is suitable for widespread clinical use to reduce markers of hyperlipidaemia.

\section{Changes in lipid metabolic proteins (CD36, PCSK9, and LDLR)}

Patients with hyperlipidaemia often exhibit abnormal expression of lipid metabolic proteins. CD36 expression was increased in patients with hypercholesterolemia [20]. A previous study showed that CD36 promoted cell uptake of free fatty acids and regulated intestinal cholesterol synthesis [21]. Additionally, ox-LDL could induce platelet activation and ROS production, ultimately resulting in thrombosis via the CD36 and MAPK pathways [22-24]. An in vivo study showed that insulin resistance and inflammatory factor release were reduced in CD36 knockout mice, indicating that CD36 plays an important role in humans [25]. CD36 is closely related to PCSK9, but in different organs, these factors behave differently. 
When PCSK9 was inhibited, CD36 expression increased in the intestine but decreased in the liver and glomerular podocytes [26]. PCSK9 could stimulate macrophages to take up ox-LDL and lead to atherosclerosis [27]. PCSK9 is a member of the proprotein invertase family of proteins, which regulate a variety of physiological and pathological processes, including lipid metabolism, inflammatory responses, glucose metabolism, apoptosis, and other processes [27]. In another report, it was found that PCSK9 might impair the function of LDLR, the LDL receptor. LDL is transported into cells via LDLR, which is closely related to the level of LDL in plasma. The deletion of the LDLR gene in familial hypercholesterolemia may be an important event in the pathogenesis of the disease [28]. A previous study showed that lipid plasmapheresis could reduce plasma PCSK9 levels [29]. Similarly, our study showed that lipid plasmapheresis could induce downregulation of the PCSK9 protein in isolated PBMCs. For the first time, the protein expression of CD36 and LDLR was shown to be obviously downregulated in PBMCs after lipid plasmapheresis. This result differs from the result obtained after therapy using statins. In patients subjected to statin therapy, PCSK9 and LDLR were upregulated, and CD36 was downregulated [30]. It is notable that LDLR was upregulated in patients using long-term statin and PCSK9 inhibitor therapy. These different results may be explained by differences in lipid clearance. The results also suggested that the removal of cholesterol and LDL from plasma by DFPP allows the body to maintain LDL levels in vivo and prevents LDL levels from dropping sharply, thus lowering LDLR and leading to a decrease in PCSK9 and CD36 levels in PBMCs. PBMCs are closely related to foam cell production, and PCSK9 and CD36 are also related to cardiovascular disease, so it is expected that lipid clearance via nondrug therapy has broad applications in the treatment of hyperlipidaemia and reduce the risk of CVD.

\section{Decrease in ER stress}

ER stress is one of the mechanisms in hyperlipidaemia. The main functions of the endoplasmic reticulum include properly folding modified proteins, controlling cholesterol production, and storing intracellular $\mathrm{Ca}^{2+}[31]$. A previous study indicated that PBMC ER stress and lipids were strongly associated with coronary artery disease [7,32]. Insults interfering with PBMC ER function lead to the accumulation of unfolded and misfolded proteins in the ER, which initiates the unfolded protein response. When the UPR fails to control the level of unfolded and misfolded proteins, ER-initiated apoptotic signalling is induced. When accompanied by high blood lipid levels, endoplasmic reticulum stress is more harmful to the body. The ER stress-related protein GRP78 was upregulated in obese animals [31]. GRP78, a signature protein of ER stress, can bind to endoplasmic reticulum stress factors to maintain the endoplasmic reticulum in a non-stress state under normal conditions [33]. Protein kinase R-like ER kinase (PERK) is the initiating protein of the endoplasmic reticulum stress pathway, and CHOP is also an important protein that links upstream and downstream proteins. For the first time, the results obtained here indicated that DFPP may be associated with a decrease in ER stress in PBMCs, indicating that lipid plasmapheresis is effective in decreasing potential risk factors for cardiovascular disease.

\section{ROS production, inflammation and apoptosis}


Hyperlipidaemia is closely associated with ROS production. Previous studies have shown that ROS is produced when endothelial like cells are exposed to free fatty acids [34]. The production of ROS has been proven to be an important cause of atherosclerosis [35]. In addition, inflammatory molecules and apoptosis-related proteins, such as IL-6, TNF-a, and BAX, were abnormally elevated in patients with hyperlipidaemia $[35,36]$. In an animal study, simvastatin administration attenuated brain oxidative stress during experimental sepsis [37]. Consistent with lipid-lowering therapy, the lipid plasmapheresis approach reported here was also associated with a decrease in ROS production, inflammation and apoptosis. It is unknown whether reducing PBMC apoptosis is beneficial in patients with hyperlipidaemia. Previous studies indicated that increases in macrophage and endothelial cell apoptosis were associated with atherosclerosis $[38,39]$. Whether the reduction in PBMC apoptosis being beneficial after lipid plasmapheresis remains to be further studied.

\section{Study strength and limitations}

DFPP is a very safe treatment means and it can reduce blood lipid levels significantly within a few hours. However, many lipid metabolic proteins changes have not been fully studied after DFPP treatment. These lipid metabolic proteins are closely related to many diseases such as CVD. In addition, ER stress, ROS production, inflammation and apoptosis were also detected in this study, which are almost related to most diseases. For the first time, it was found that the expression of lipid metabolism-related proteins and endoplasmic reticulum stress-related proteins was reduced, and the levels of ROS were also reduced in PBMCs. The results demonstrated that DFPP has many protective effects on the human body in addition to reducing lipids, and it can be used as a means to treat many diseases in the future. The development of membrane plasma component separator materials can make DFPP as a effective means to treat many diseases in the future.

There are several potential limitations in study. First, lipid metabolism- and ER stress-related proteins were not continuously measured after lipid plasmapheresis, and data concerning when plasma purification treatment should be repeated was not obtained. Second, there are many lipid-related proteins; some lipidrelated proteins were not measured. So it is necessary to assay all lipid metabolism-related proteins to provide clinicians with complete information about lipid plasmapheresis.

\section{Conclusion}

The results of this study demonstrated that DFPP reduced the levels of lipids, lipid-metabolizing proteins, and inflammatory factors in patients with hyperlipidaemia. It also reduced the levels of endoplasmic reticulum stress- and apoptosis-related proteins and ROS in PBMCs. Plasma purification shows promise for the treatment of patients with hyperlipidaemia. Lipid plasmapheresis is also inexpensive, easy to perform, safe and effective.

\section{Declarations}


Acknowledgements

Not applicable.

\section{Authors' contributions}

Yang $\mathrm{XH}$ and Jin HM conceived of and designed the research. All authors conducted experiments and analyzed data. Zhang XM, Gu YH, Zhong ZY, Xu ZQ and Deng H wrote the manuscript, and all authors commented on previous versions of the manuscript. All authors read and approved the final manuscript. Zhang XM, Gu YH and Deng H contribute equally to this paper.

Funding: This study was supported by Program of Pudong Hospital (YJ2020-04), Discipline Construction Promoting Project of Shanghai Pudong Hospital in Nephrology (Zdxk2020-10), Plasma Purification (Zdzk2020-12) and Shanghai Municipal Health Commission (Grant No. 201840025).

\section{Availability of data and materials}

The datasets generated during and/or analyzed during the current study are available from the corresponding author upon reasonable request.

\section{Ethics approval and consent to participate}

All patients provided and signed informed consents. The study was approved by the Ethical Committee of Shanghai Pudong Hospital (Fudan University, Pudong Medical Center, Shanghai, China) and experiments were performed in accordance with the Ethical Committee's guidelines and regulations. ClinicalTrials.gov number was NCT 03491956.

\section{Consent for publication}

All authors have approved the final version of the manuscript and have agreed to submit it to this journal.

\section{Competing interests}

The authors declare no conflict of interest with respect to the publication of this article.

\section{Abbreviations}

CVD: Cardiovascular disease

DFPP: Double-filtration plasmapheresis

ER: Endoplasmic reticulum

PBMCs: Peripheral blood mononuclear cells

PCSK9: Proprotein convertase subtilisin/kexin type 9 
GRP78:Glucose-regulated protein 78

CHOP:C/EBP homoiogousprotein

ATF4:Activating transcription factor 4

EIF2:Eukaryotic initiation factor 2

ROS:Reactive oxygen species

Lp (a):lipoprotein a

LDLR:LDL receptor

HELP:Heparin-induced extracorporeal LDL precipitation

PVDF: Transferred onto polyvinylidene difluoride

SDS-PAGE:Sodium dodecyl sulfate-polyacrylamide gel electrophoresis

PERK:Protein kinase R-like ER kinase

IL-1b:Interleukin 1b

IL-6:Interleukin 6

TNF-a:Tumor necrosis factor a

\section{References}

1. Morgan PK, Fang L, Lancaster GI, Murphy AJ. Hematopoiesis is regulated by cholesterol efflux pathways and lipid rafts: connections with cardiovascular diseases. J Lipid Res. 2019; jlr.TR119000267. [Epub ahead of print]

2. McCully KS. Chemical Pathology of Homocysteine VII. Cholesterol, Thioretinaco Ozonide, Mitochondrial Dysfunction, and Prevention of Mortality. Ann Clin Lab Sci. 2019;49: 425-438.

3. Ozkok A. Cholesterol-embolization syndrome: current perspectives. Vasc Health Risk Manag. 2019;15: 209-220.

4. Püschel GP, Henkel J. 2018. Dietary cholesterol does not break your heart but kills your liver. Porto Biomed J. 2019;3 (1):e12.

5. Guo YL, Li J. Interpretation of guideines for the prevention and treatment of dyslipidemia in Chinese adults (2016 revision). Chinese J. Front Med Sci. 2016; 9(6)12-14.

6. Couturier J, Nuotio-Antar AM, Agarwal N, Wilkerson GKロSaha P, Kulkarni V, Lakhashe SK, Esquivel J, Nehete PN, Ruprecht RM,Sastry KJ, Meyer JM, Hill LR, Lake JE, Balasubramanyam A, Lewis DE. Lymphocytes upregulate CD36 in adipose tissue and liver. Adipocyte. 2019;8(1):154-163. 
7. Park, Y. M. CD36, a scavenger receptor implicated in atherosclerosis. Exp Mol Med. 2014; 46(6):e99.

8. Raghavan S, Singh NK, Gali S, Mani AM, Rao GN. Protein Kinase $C \theta$ Via Activating Transcription Factor 2-Mediated CD36 Expression and Foam

Cell Formation of Ly6C ${ }^{\text {hi }}$ Cells Contributes to Atherosclerosis. Circulation. 2018;138: 2395-2412.

9. Cohen JC, Boerwinkle E, Mosley TH Jr, Hobbs HH. Sequence variations in PCSK9, low LDL, and protection against coronary heart disease. N Engl J Med. 2006;354 (12):1264-1272.

10. McNutt MC, Lagace TA, Horton JD. Catalytic activity is not required for secreted PCSK9 to reduce low density lipoprotein receptors in HepG2 cells. J Biol Chem. 2007;Jul 20;282):20799-803.

11. Sinnaeve PR, Schwartz GG, Wojdyla DM, Alings M, Bhatt DL, Bittner VA, Chiang CE, Correa Flores RM, Diaz R, Dorobantu M,Goodman SG, Jukema JW, Kim YU, Pordy R, Roe MT, Sy RG, Szarek $M$, White HD, Zeiher AM, Steg PG; ODYSSEY OUTCOMES Investigators. Effect of alirocumab on cardiovascular outcomes after acute coronary syndromes according to age: an ODYSSEY OUTCOMES trial analysis. Eur Heart J. 2019;ehz809. [Epub ahead of print]

12. Baiceanu A, Mesdom P, Lagouge M, Foufelle F. Endoplasmic reticulum proteostasis in hepatic steatosis. Nat Rev Endocrinol. 2016;12(12):710-722.

13. Rajpathak SN, Kumbhani DJ, Crandall J, Barzilai N, Alderman M, Ridker PM. Statin therapy and risk of developing type 2 diabetes: a meta-analysis. Diabetes Care. 2009;32:1924-9.

14. 14. https://www.drugs.com/pro/ezetimibe.html\#s-34084-4

15. Tsai JL, Wu MJ, Shu KH, Tsai SF. Long-Term Follow-Up of a Homozygous Familial Hypercholesterolemic Patient Receiving Regular Double Filtration Plasmapheresis-Case Report and Literature Review. Blood Purif. 2016;41(4):264-269.

16. Taylan C, Schlune A, Meissner T, Ažukaitis K, Udink Ten Cate FE, Weber LT. Disease control via intensified lipoprotein apheresis in three siblings with familial hypercholesterolemia. J Clin Lipidol. 2016;10(6):1303-1310.

17. Chang CT, Tsai TY, Liao HY, Chang CM, Jheng JS, Huang WH, Chou CY, Chen CJ. Double Filtration Plasma Apheresis Shortens Hospital Admission Duration of Patients With Severe Hypertriglyceridemia-Associated Acute Pancreatitis. Pancreas. 2016;45(4):606-612.

18. Tishko VV, Sokolov AA, Belskih AN, Ivanov AM, Meshkova ME, Skorinova TS. Impact of double filtration plasmapheresis on adhesion molecules levels in patients with stable coronary heart disease after coronary stenting. Atheroscler Suppl. 2017; 30: 92-98.

19. Yang XH, Pan Y, Zhan XL, Zhang BL, Guo LL, Jin HM. Epigallocatechin-3-gallate Attenuates Renal Damage by Suppressing Oxidative Stress in Diabetic db/db Mice. Oxid Med Cell Longev. 2016;2968462. Epub 2016 Sep 6.

20. Yazgan B, Sozen E, Karademir B, Ustunsoy S, Ince U, Zarkovic N, Ozer NK. CD36 expression in peripheral blood mononuclear cells reflects the onset of atherosclerosis. Biofactor. 2018;44(6):588596. 
21. Nassir F, Wilson B, Han X, Gross RW, Abumrad NA. CD36 is important for fatty acid and cholesterol uptake by the proximal but not distal intestine. J Biol Chem. 2007; 282(27):19493-501.

22. Yang M, Cooley BC, Li W, Chen Y, Vasquez-Vivar J, Scoggins NO, Cameron SJ, Morrell CN, Silverstein RL. Platelet CD36 promotes thrombosis by activating redox sensor ERK5 in hyperlipidemic conditions. Blood. 2017; 129(21):2917-2927.

23. Magwenzi S, Woodward C, Wraith KS, Aburima A, Raslan Z, Jones H, McNeil C, Wheatcroft S, Yuldasheva N, Febbriao M, Kearney M, Naseem KM. Oxidized LDL activates blood platelets through CD36/NOX2-mediated inhibition of the cGMP/protein kinase G signaling cascade. Blood. 2015; 125(17):2693-2703.

24. Podrez EA, Byzova TV, Febbraio M, Salomon RG, Ma Y, Valiyaveettil M, Poliakov E, Sun M, Finton PJ, Curtis BR, Chen J, Zhang R, Silverstein RL, Hazen SL. Platelet CD36 links hyperlipidemia, oxidant stress and a prothrombotic phenotype. Nat Med. 2007;13(9):1086-1095.

25. Kennedy DJ, Kuchibhotla S, Westfall KM, Silverstein RL, Morton RE, Febbraio M. 2011. A CD36dependent pathway enhances macrophage and adipose tissue inflammation and impairs insulin signalling. Cardiovasc Res. 89(3):604-613.

26. Dixon DL, Trankle C, Buckley L, Parod E, Carbone S, Van Tassell BW, Abbate A. A review of PCSK9 inhibition and its effects beyond LDL receptors. J Clin Lipidol. 2016;10(5):1073-1080.

27. Ding Z, Liu S, Wang X, Theus S, Deng X, Fan Y, Zhou S, Mehta JL. PCSK9 regulates expression of scavenger receptors and ox-LDL uptake in macrophages. Cardiovasc Res. 2018;114(8):1145-1153.

28. Benito-Vicente A, Uribe KB, Jebari S, Galicia-Garcia U, Ostolaza H, Martin C. Validation of LDLr Activity as a Tool to Improve Genetic Diagnosis of Familial Hypercholesterolemia: A Retrospective on Functional Characterization of LDLr Variants. Int J Mol Sci. 2018;5;19(6).

29. Julius U, Milton M, Stoellner D, Rader D, Gordon B, Polk D, Waldmann E, Parhofer KG, Moriarty PM. Effects of lipoprotein apheresis on PCSK9 levels. Atheroscler Suppl. 2015;18: 180-186.

30. Nozue T. Lipid Lowering Therapy and Circulating PCSK9 Concentration. J Atheroscler Thromb. 2017;24(9):895-907.

31. Ozcan U, Cao Q, Yilmaz E, Lee AH, Iwakoshi NN, Ozdelen E, Tuncman G, Görgün C, Glimcher LH, Hotamisligil GS. Endoplasmic reticulum stress links obesity, insulin action, and type 2 diabetes. Science. 2004;306(5695):457-461.

32. Mozzini C, Fratta Pasini A, Garbin U, Stranieri C, Pasini A, Vallerio P, Cominacini L. Increased endoplasmic reticulum stress and Nrf2 repression in peripheral blood mononuclear cells of patients with stable coronary artery disease. Free Radic Biol Med.2014;68:178-85.

33. Lee AS. The ER chaperone and signaling regulator GRP78/BiP as a monitor of endoplasmic reticulum stress. Methods. 2005;35(4):373-81.

34. Storniolo CE, Roselló-Catafau J, Pintó X, Mitjavila MT, Moreno JJ. Polyphenol fraction of extra virgin olive oil protects against endothelial dysfunction induced by high glucose and free fatty acids through modulation of nitric oxide and endothelin-1. Redox Biol. 2014;2:971-7. 
35. Stocker R, Keaney JF Jr. Role of oxidative modifications in atherosclerosis. Physiol Rev. 2004;84(4):1381-1478.

36. Stefanutti C, Mazza F, Pasqualetti D, Di Giacomo S, Watts GF, Massari MS, de Neve J, Morozzi C, Fischer M. Lipoprotein apheresis downregulates IL-1a, IL-6 and TNF-a mRNA expression in severe dyslipidaemia. Atheroscler Supp. 2017;30: 200-208.

37. Catalão CHR, Santos-Júnior NN, da Costa LHA, Souza AO, Alberici LC, Rocha MJA. Brain Oxidative Stress During Experimental Sepsis Is Attenuated by Simvastatin Administration. Mol Neurobiol. 2017; 54(9):7008-7018.

38. Linton MF, Moslehi JJ, Babaev VR. Akt Signaling in Macrophage Polarization, Survival, and Atherosclerosis. Int J Mol Sci. 2019;20(11):2703.

39. Paone S, Baxter AA, Hulett MD, Poon IKH. Endothelial cell apoptosis and the role of endothelial cellderived extracellular vesicles in the progression of atherosclerosis. Cell Mol Life Sci. 2019;76 (6):1093-1106.

\section{Tables}

Table 1 The variations of lipids before and after DFPP treatment.

\begin{tabular}{|lll|}
\hline & Pre-DFPP $(\mathrm{N}=35)$ & Post-DFPP $(\mathrm{N}=35)$ \\
\hline Age $($ years $)$ & $51[44,61]$ & - \\
\hline Male & 12 & - \\
\hline Weight $(\mathrm{kg})$ & $73.1[68.4,83.9]$ & - \\
\hline Total cholesterol $(\mathrm{mM})$ & $8.2[6.6,10.0]$ & $2.4[1.8,3.1]^{\star \star}$ \\
\hline Triglyceride $(\mathrm{mM})$ & $8.9[7.1,10.2]$ & $2.1[1.5,3.1]^{\star \star}$ \\
\hline LDL-C $(\mathrm{mM})$ & $4.7[3.9,5.5]$ & $1.4[1.1,1.8]^{\star \star}$ \\
\hline Lp(a) $(\mathrm{mg} / \mathrm{L})$ & $187.3[137.9,200.7]$ & $78.3[53.8,104.8]^{\star \star}$ \\
\hline sdLDL-C $(\mathrm{mM})$ & $2.1[1.8,2.7]$ & $0.3[0.2,0.5]^{\star \star}$ \\
\hline Patients with hypercholesterolemia & 18 & - \\
\hline Patients with hypertriglyceridemia & 10 & - \\
\hline Patients with mixed hyperlipidemia & 3 & - \\
\hline Patients with high low-density lipoproteinemia & 4 & - \\
\hline
\end{tabular}

Pre-DFPP: patients before DFPP treatment; Post-DFPP: patients after DFPP treatment; LDL-C, low density lipoprotein; $L p(a)$, lipoprotein(a). sdLDL, small dense low-density lipoprotein. Data were expressed as median (range). ${ }^{* *} P<0.01$ vs pre-DFPP. 
Table 2 DFPP reduces inflammation parameters

\begin{tabular}{|lll|}
\hline & Pre-DFPP $(\mathrm{N}=35)$ & Post-DFPP $(\mathrm{N}=35)$ \\
\hline $\mathrm{IL}-1 \beta(\mathrm{pg} / \mathrm{mL})$ & $17.9[15.3,20.1]$ & $9.3[8.1,10.7]^{\star}$ \\
\hline $\mathrm{IL}-6(\mathrm{pg} / \mathrm{mL})$ & $16.0[13.4,20.8]$ & $5.6[4.1,8.4]^{\star \star}$ \\
\hline $\mathrm{TNF}-\mathrm{a}(\mathrm{pg} / \mathrm{mL})$ & $21.7[19.5,23.5]$ & $9.3[8.5,11.0]^{\star \star}$ \\
\hline
\end{tabular}

Pre-DFPP: patients before DFPP treatment; Post-DFPP: patients after DFPP treatment; Serum IL-6, TNF-a, IL-1 $\beta$ in patients with hyperlipidemia were detected by ELISA kit. Data were expressed as median (range). $\star P<0.05$ and ${ }^{*} P<0.01$ vs. Pre-DFPP.

\section{Figures}

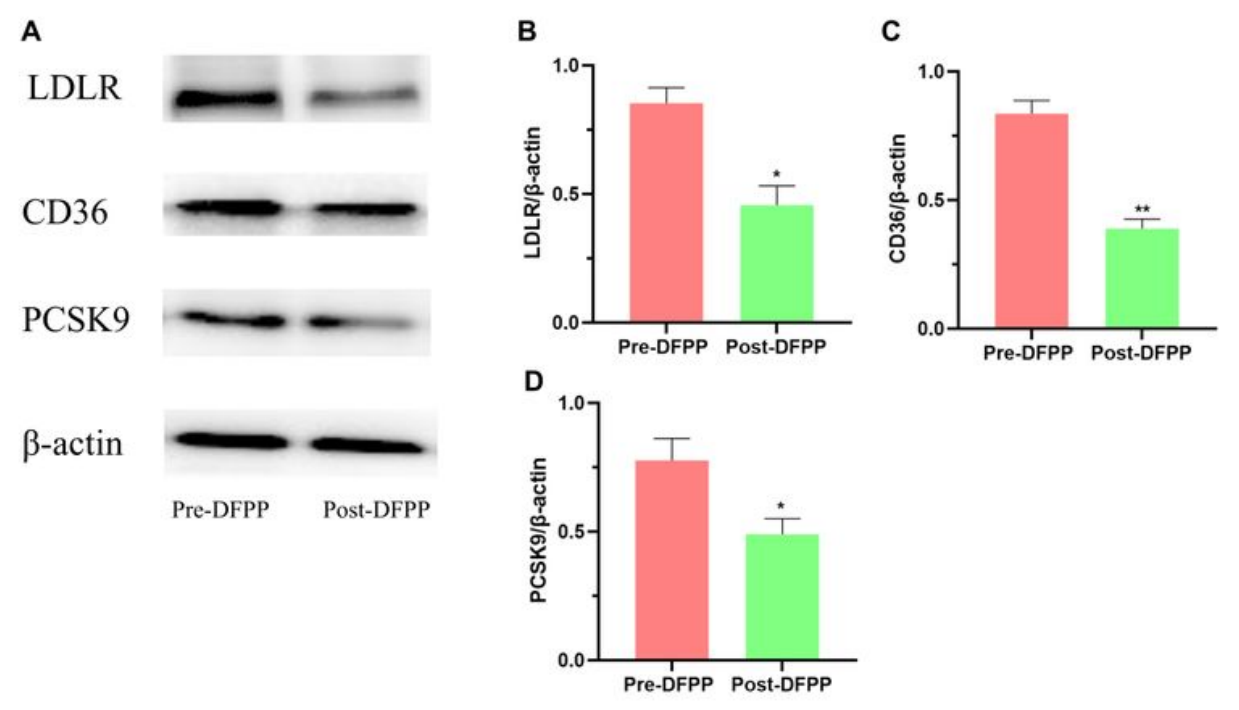

\section{Figure 1}

Effects of DFPP on expressions of lipid metabolic proteins The protein expression of LDLR (B), CD36 (C), PCSK9 (D) in PBMCs were detected by Western blot analyses. Pre-DFPP: patients before DFPP. PostDFPP: patients post DFPP. Values were means \pm SD. ${ }^{*}<0.05$ and ${ }^{*} P<<0.01$ vs. Pre-DFPP. $N=5$ 

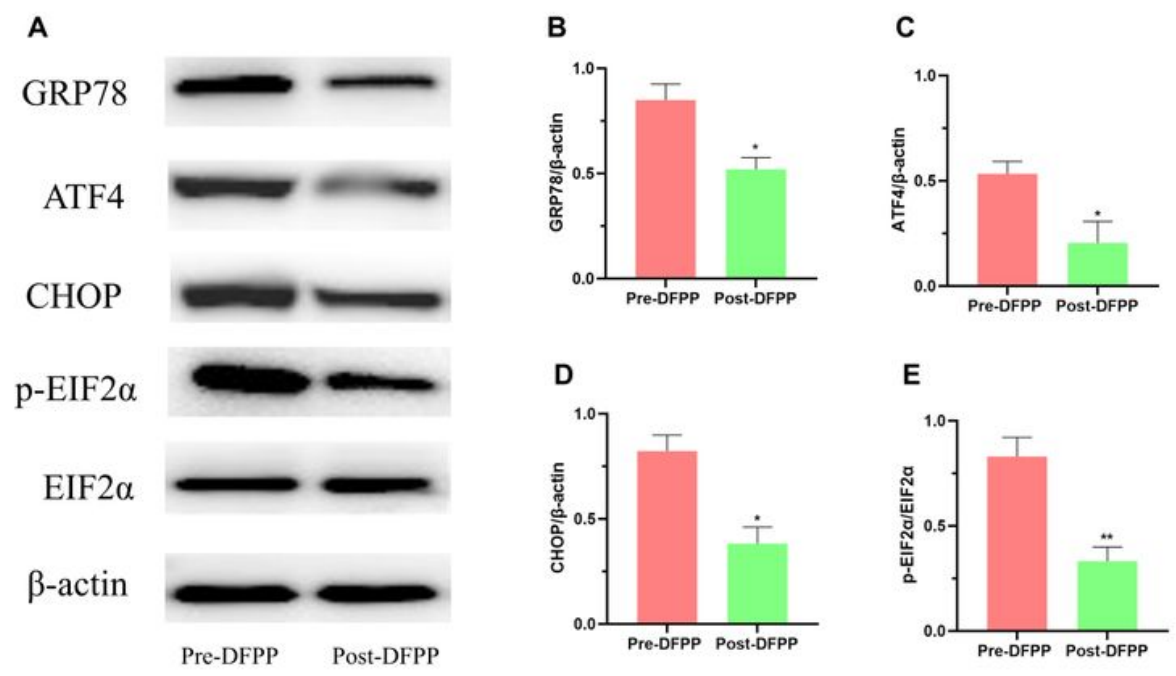

\section{Figure 2}

DFPP affects ER stress-related proteins ER stress-related proteins [GRP78 (B), ATF4 (C), CHOP (D), pEIF2a (E)] in PBMCs were detected by Western blot analyses. Values were means $\pm S D$. ${ }^{*}<0.05$ and $\star \star P<0.01$ vs. Pre-DFPP. $N=5$ 


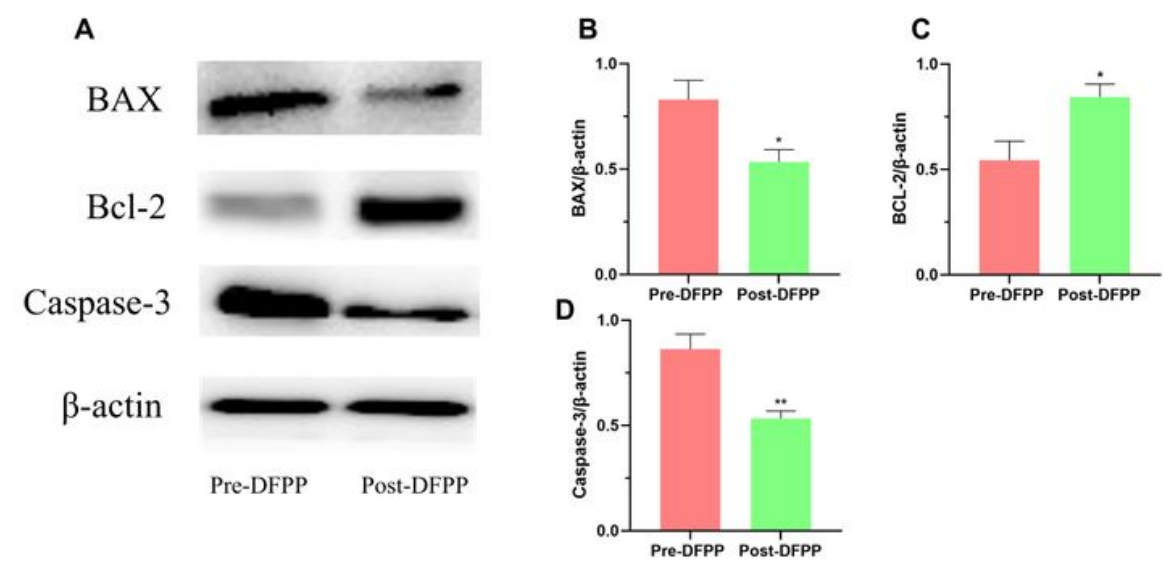

\section{Figure 3}

Effects of lipid plasmapheresis on expressions of apoptosis-related proteins Apoptosis-related proteins $[\mathrm{Bcl}-2$ (B) $\triangle \mathrm{BAX}(\mathrm{C}) \otimes C a s p a s e-3(\mathrm{D})]$ in PBMCs were examined by Western blotting analyses. Values were means $\pm S D$. ${ }^{*} P<0.05$ and ${ }^{*} \times<<0.01$ vs. Pre-DFPP. $N=5$ 

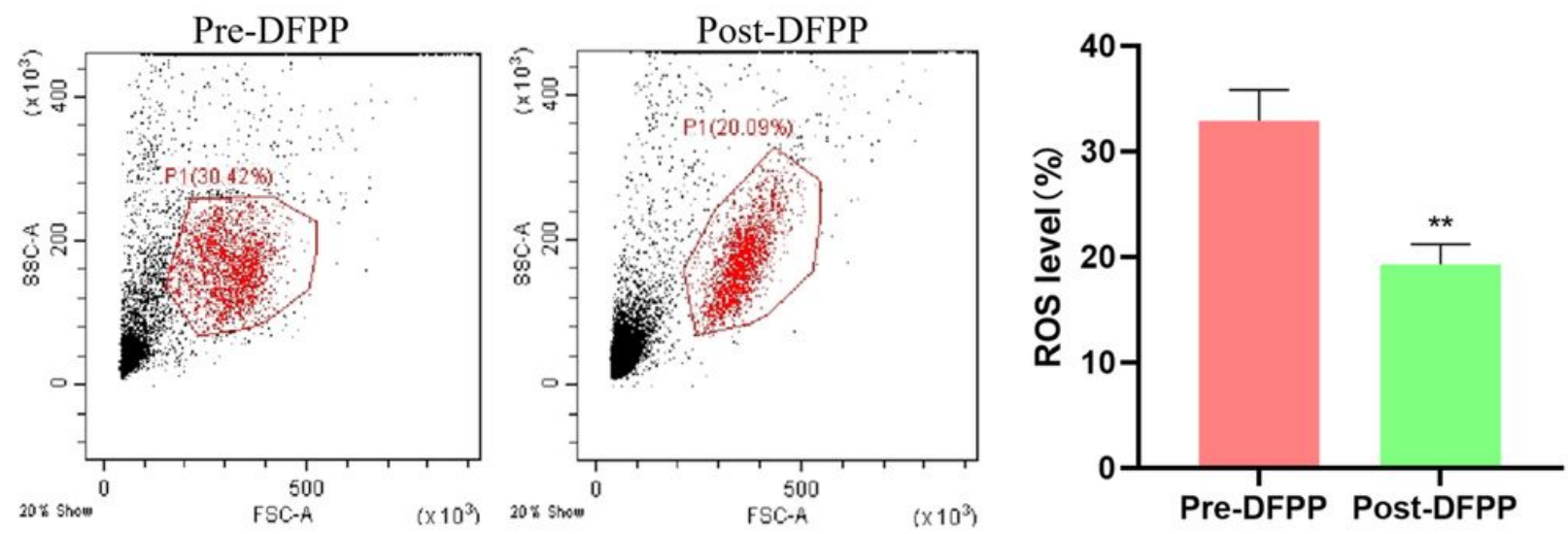

\section{Figure 4}

DFPP reduces ROS level ROS level in PBMCs was examined by flow cytometry. Values were means \pm SD. $\star * P<0.01$ vs. Pre-DFPP. $N=5$ 